\title{
Characteristics of femoroacetabular impingement morphology and relation with skeletal maturity among asymptomatic adolescents
}

\author{
Mehmet Kaymakoglu, Raziye Dut, Duygu Imre, Fatma Bilge Ergen, Mehmet Ali Talmac, Cemalettin Aksoy
}

From the Department of Orthopaedics, Traumatology and Radiology, Hacettepe University, Ankara, Turkey

The aim of this study was to analyze presence of the morphological characteristics and prevalence of FAI in asymptomatic adolescents and assess the relation of skeletal maturation with development of FAI morphology.

Abdominopelvic computed tomography (CT) of 265 adolescents (9-19 years old) who were admitted to the emergency department between 2011 and 2016 were evaluated retrospectively. Radial reformatted CT images from the femoral neck were created using the multiplanar reconstruction (MPR) method. The femoral neck was divided into 12 segments and alpha angle (AA), femoral head-neck ratio (FHNR) and center-edge angle (CEA) were measured from each segment. Additionally, images were evaluated for the physiological status (open or closed) of the triradiatecartilage and proximal femoral epiphyses.

204 hips from 102 patients (32 females, 70 males) were retrospectively reviewed. There were 27 (26.5\%) patients with cam-type morphology and 18 $(17.6 \%)$ patients with pincer-type morphologies. No statistically significant difference was detected between the prevalences of cam and pincer morphologies between the two genders. Cam deformity was most frequently seen in anterosuperior segment. All of the patients $(100 \%)$ with pincer-type morphology and $88 \%$ of the patients with cam-type morphology had closed triradiate cartilage, $89 \%$ of the patients with cam morphology and $83 \%$ with pincer morphology had open proximal femoral physis.

Our results showed that prevalence of cam and pincer-type morphology in asymptomatic adolescents is similar to asymptomatic adults. Our findings also

No benefits or funds were received in support of this study. None of the authors have a conflict of interest. indicate that cam- and pincer-type FAI morphologies likely develop during late adolescence after closure of triradiate cartilage and before closure of proximal femoral physis.

Level of Evidence - 3

Keywords : femoroacetabular impingement; cam ; pincer ; physeal closure.

Mehmet Kaymakoglu, ${ }^{1,2}$, MD,

Raziye Dut ${ }^{3}, \mathrm{MD}$,

- Duygu Imre ${ }^{4}, \mathrm{MD}$,

- Fatma Bilge Ergen ${ }^{4}, \mathrm{MD}$,

- Mehmet Ali Talmac ${ }^{5}, \mathrm{MD}$,

- Cemalettin Aksoy ${ }^{1}, \mathrm{MD}$

${ }^{1}$ Department of Orthopaedics and Traumatology, Hacettepe University, Ankara, Turkey.

${ }^{2}$ Department of Orthopaedics and Traumatology, Bornova Turkan Ozilhan State Hospital, Izmir, Turkey.

${ }^{3}$ Department of Paediatrics, Hacettepe University, Ankara, Turkey.

${ }^{4}$ Department of Radiology, Hacettepe University, Ankara, Turkey.

${ }^{5}$ Department of Orthopaedics and Traumatology, Sisli Etfal

Hamidiye Research Hospital, Istanbul, Turkey.

Correspondence : Cemalettin Aksoy, Hacettepe University, Department of Orthopaedics and Traumatology, Sihhiye, 06100 Ankara, Turkey. Phone : +90 5322310076.

Email : aksoyc@hotmail.com

Fatma Bilge Ergen, Hacettepe University, Department of Radiology, Sihhiye, 06100 Ankara, Turkey. Phone : +90 532 7138016 .

Email : bergen@delta-eur.com

- 2021, Acta Orthopædica Belgica. 


\section{INTRODUCTION}

A variety of hip joint deformities which thought to contribute to the development of osteoarthritis have been reported, but to date, no satisfactory causative relation is found. There is an increasing evidence that abnormal anatomy of the femoral head-neck junction and/or acetabulum without dysplasia are the major etiologic factors in the pathogenesis of hip osteoarthritis. (1-4). Whether these minor changes can cause primary hip osteoarthritis has remained unclear until Ganz et al. introduced the term 'femoroacetabular impingement (FAI)' in 2003 (5). Although it does not enlighten the etiology of a significant group of young patients with hip osteoarthritis, the FAI theory was widely accepted by many authors as a major cause of 'primary' hip osteoarthritis. The following three types of FAI has been described; acetabular overgrowth causing an over-coverage of the femoral head (pincer type), an excessive bony hump at the femoral head-neck junction causing an aspherical shape and 'pistolgrip' deformity (cam type), and a mixed type of both forms. The abnormal contact of the femoral head and acetabulum may damage the labrum and cartilage over time ; thus, the early onset of hip osteoarthritis develops $(5,6)$.

Many theories have been considered in the development of FAI morphology, including migration, microinstability, genetic factors, repetitive trauma, slipped capital femoral epiphysis, and Legg-Calve-Perthes disease (7-10). In addition to the absence of the definite explanation about its etiology, it is still not known whether hips with FAI morphology eventually resulted in development of osteoarthritis. Epidemiological studies reported that the prevalence of FAI morphology is not rare, varying from $10 \%$ to $74 \%$, which suggests that it is an anatomical variant rather than a pathological condition. Patients who are asymptomatic but have FAI morphology are candidates for development of osteoarthritis (5). Determination of which patients could develop osteoarthritis in the future is still a matter of debate in today's literature.

This study aimed to analyze the prevalence of the FAI morphology among asymptomatic adolescents and determine normative measurements in this patient population. We also aimed to assess the relation of skeletal maturation in the development of FAI morphology by assessing closure of the triradiate cartilage and proximal femoral physis.

\section{MATERIALS AND METHODS}

After obtaining the ethics committee approval from our institution, we retrospectively reviewed abdominopelvic computed tomography (CT) images of 265 adolescents aged between 9 and 19 years who underwent abdominopelvic $\mathrm{CT}$ for the reasons other than hip problems. They were admitted to the emergency department of our hospital between 2011 and 2016, which meets a heavy demand of patient population in its region. Patients' medical data were reviewed and we excluded patients with hip pain and hip and spine related conditions including, fracture, bone tumor, previous surgery, history of developmental dysplasia of the hip, metabolic, or genetic diseases, and gait disturbances.

\section{Image analysis}

Computed tomography measurements were performed using one CT scanner (Siemens SOMATOM Definition AS, Siemens Healthcare, Erlangen, Germany). We only included patients who had thin slice CT images $(\leq 1 \mathrm{~mm})$ since thicker slices were not suitable for MPR reconstruction.

After applying the exclusion criteria, 102 patients (204 hips) were left for the evaluation. Axial CT images were transferred from the Picture Archiving and Communicating System (PACS) (Centricity PACS-IW 3.7.3, GE Healthcare, Milwaukee, Wisconsin, USA) to the Siemens Workstation and 2D radial reformatted images were created with MPR technique, as it is the gold standard method in evaluating the AA at the femoral head-neck junction (11).

The first 30 out of 204 hips, radial reformatted images and all measurements were performed by two observers (M.K respectively) under the supervision of an experienced musculoskeletal radiologist (F.B.E). The rest of the measurements were done by the two observers (D.I and independently). In randomly selected 30 of patients 
all quantitative parameters were measured by two observers to assess intraobserver variability.

Twelve $\left(\mathrm{AA}_{1}-\mathrm{AA}_{12}\right)$ axial oblique MPR images were reconstructed in $30^{\circ}$ intervals. $\mathrm{AA}_{12}$ being the most superior, $\mathrm{AA}_{3}$ the most anterior, $\mathrm{AA}_{6}$ the most inferior, and $\mathrm{AA}_{9}$ the most posterior. $\mathrm{AA}$ was measured with Nötzli's method from each segment (12). For AA measurement, a circle was drawn around the margin of the femoral head. The point where the distance from the center of the head exceeds the radius and crosses the circle was chosen to create an angle with a line which is parallel to the femoral neck (Figure 1). We accepted a threshold of $>55^{\circ}$ degrees for the presence of cam-type morphology as the current literature accepts this cutoff value in younger patients (13).

FHNR was measured in axial plane as described by Murray (1) (Figure 2). For FHNR measurement, two parallel lines were drawn which are tangential to the anterior margins of femoral head and neck. The distances from the axis of the femoral neck perpendicular to these two lines were measured (Figure 2, a and b). The difference between the distances perpendicular to these lines were divided into the radius of the femoral head. As described by Eijer et al (14) we accepted a threshold of 0.13 for the presence of cam-type morphology.

Center-edge angle (CEA) was used for the assessment of the pincer-type morphology. Two lines were drawn from the center of the femoral

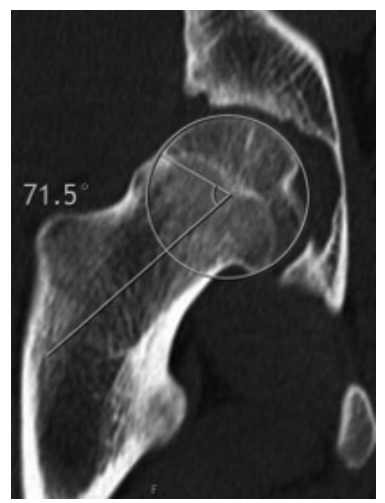

Figure 1. - Measurement technique of AA as described by Nötzli. A circle was drawn around the margin of the femoral head. The point where the distance from the center of the head exceeds the radius and crosses the circle was chosen to create an angle with a line which is parallel to the femoral neck.



Figure 2. - Measurement technique of FHNR. Two parallel lines were drawn which are tangential to the anterior margins of femoral head and neck. The distances from the axis of the femoral neck perpendicular to these two lines were measured ( $a$ and $r$ ). The difference between the distances perpendicular to these lines were divided into the radius of the femoral head $(\mathrm{r}-\mathrm{a} / \mathrm{r})$.

head; one passing from the center of the femoral head towards the most lateral margin of the acetabulum and the other is a vertical reference line, which was determined perpendicular to the center of the femoral head. We accepted a threshold of $\geq$ $40^{\circ}$ for the presence of pincer-type morphology.

A patient who has $\mathrm{AA} \geq 55^{\circ}$ or $\mathrm{FHNR}<0.13$ was accepted to have a cam-type and CEA $\geq 40^{\circ}$ as pincer-type morphology $(12,15)$. Patients who has $\mathrm{AA} \geq 55^{\circ}$ or $\mathrm{FHNR}<0.13$ and $\mathrm{CEA}>40^{\circ}$, was accepted as mixed-type morphology.

The physiological status of the proximal femoral physis and triradiate cartilage were also assessed by two observers (D.I and M.K) with consensus. If there was a full fusion of two adjacent bones throughout the length of the triradiate cartilage and proximal femoral physis on every slice of CT images that involves the physeal line, it was called 'closed', and, if there was a gap between two adjacent bones throughout the length of the physis in at least 2 sequential slices it was called as 'open' (Figure 3) (16). Same criteria was applied for the assessment of proximal femoral physis. 

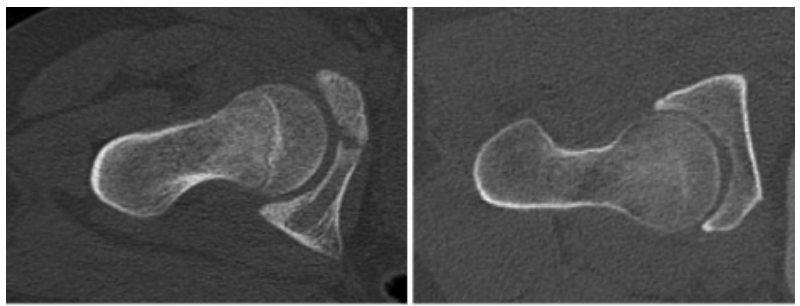

Figure 3. - Status of physeal lines. CT-image of a 12 year old boy whose proximal femoral physis and triradiate cartilage is open (A). Closed proximal femoral physis and triradiate cartilage on a CT-image of a 17 year old girl (B).

\section{Statistical analysis}

The prevalence of cam and pincer deformities between the two sexes was compared using the student's-t test and Mann-Whitney U test. The difference of mean AA value between two sexes of all segments $\left(\mathrm{A}_{1-12}\right)$ and mean $\mathrm{AA}$ value at the $\mathrm{AA}_{2}$ were compared using t-test.

IBM SPSS Statistics v. 23.0 was used for the statistics and $P$ value was set at $P=0.05$ for significance.

\section{RESULTS}

Two hundered and four hips in 102 patients ( 32 females, 70 males) were retrospectively reviewed. The mean age of the patients were 14.8 (range 9-19, SD : 3.0) years. The mean AA was $40^{\circ}$ (range $28^{\circ}$ $78^{\circ}$, SD : 5.3, CI : 39.3-39.7), and the mean CEA was $32^{\circ}$ (range $22^{\circ}-46^{\circ}$, SD : 6.7, CI : 31.2-33.0). Twenty-seven patients $(26.5 \%)$ had an AA $\geq 55^{\circ}$ and $18(17.6 \%)$ patients had a $C E A \geq 40^{\circ}$. The mean FHNR was 0.15 (range 0.1-0.27, SD : 0.03). (Table I).

Twenty-seven of the 102 (24.5\%) patients (55 of the 204 hips, 26.9\%) had cam-type morphology and $10(42 \%)$ were unilateral. Cam-type morphology

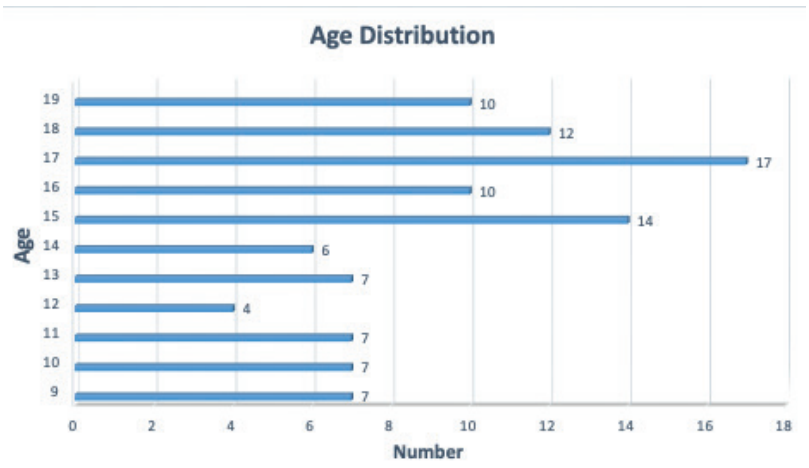

Figure 4. - Age distribution of the patient population.

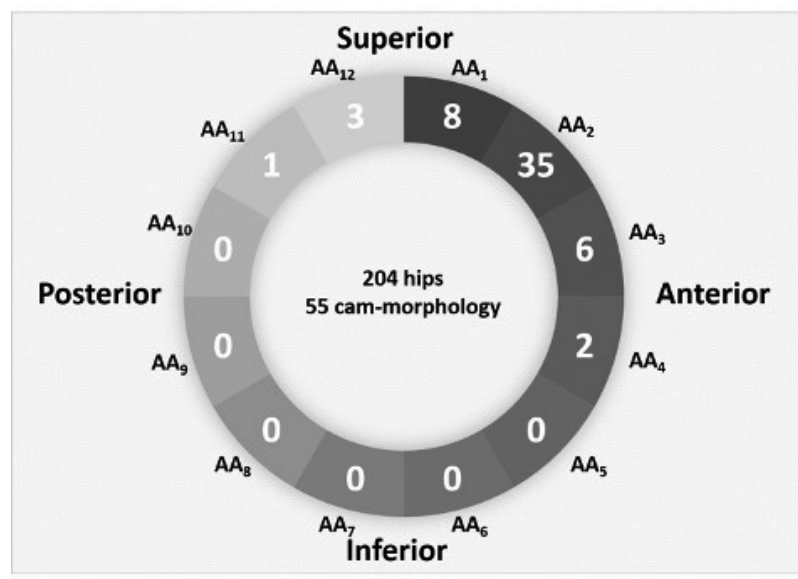

Figure 5. - Distribution of the location of cam deformities at the femoral head-neck junction.

was more common in males $(18 / 70 ; 28.6 \%)$ than females $(7 / 32 ; 21.9 \%)$, but there was no statistically significant difference between the two sexes $(P=0.34)$.

The most prevalent segment for cam deformity was $\mathrm{AA}_{2}$, which is the anterosuperior segment of the femoral neck (1-2 o'clock). Cam deformities were observed in 9 of the 204 hips at $\mathrm{AA}_{1}$ (121 o'clock), 36 hips at $\mathrm{AA}_{2}$ (1-2 o'clock), 6 hips at $\mathrm{AA}_{3}$ (2-3 o'clock), 2 hips at $\mathrm{AA}_{4}$ (3-4 o'clock),

Table I. - AA and CEA values and deformity rates in both genders

\begin{tabular}{|l|c|c|c|c|}
\hline & Alpha Angle (SD) & Cam deformity (\%) & CEA angle (SD) & Pincer deformity (\%) \\
\hline Males & $40^{\circ} \pm(5.63)$ & $20 / 70(28.5 \%)$ & $32^{\circ} \pm(6.19)$ & $11 / 70(15.7 \%)$ \\
\hline Females & $39^{\circ} \pm(5.19)$ & $7 / 32(21.9 \%)$ & $32^{\circ} \pm(5.88)$ & $7 / 32(21.9 \%)$ \\
\hline Total & $40^{\circ} \pm(5.38)$ & $27 / 102(26.5 \%)$ & $32^{\circ} \pm(6.79)$ & $18 / 102(17.6 \%)$ \\
\hline
\end{tabular}

SD: Standard deviation 


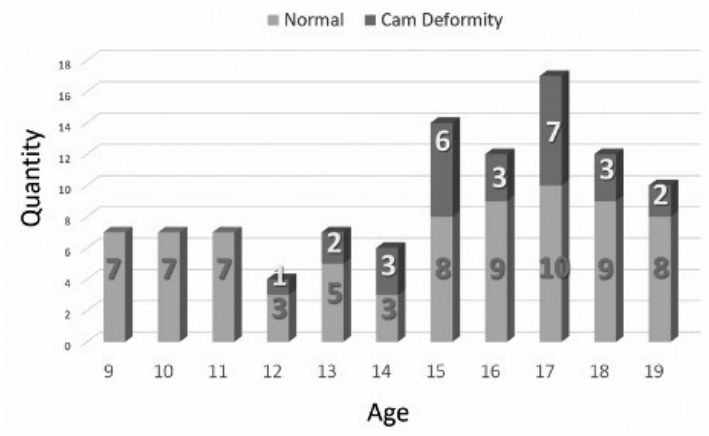

Figure 6. - Distribution of patients with cam-morphology according to each age groups.



Figure 7. - Distribution of pincer-type morphology according to age.

Table II. - Physiological status of triradiate cartilage and proximal femoral physis in FAI-morphological patients. (PFP : proximal femoral physis)

\begin{tabular}{|l|c|c|c|c|}
\hline & Open triradiate (n) & Closed triradiate (n) & Open PFP (n) & Closed PFP (n) \\
\hline Pincer morphology $(\mathbf{n}=\mathbf{1 8})$ & $0(0 \%)$ & $18(100 \%)$ & $15(83 \%)$ & $3(17 \%)$ \\
\hline Cam morphology $(\mathbf{n}=\mathbf{2 7})$ & $3(11 \%)$ & $24(89 \%)$ & $24(89 \%)$ & $3(11 \%)$ \\
\hline Mix morphology $(\mathbf{n}=\mathbf{5})$ & $0(0 \%)$ & $5(100 \%)$ & $5(100 \%)$ & $0(0 \%)$ \\
\hline
\end{tabular}

1 hip at $\mathrm{AA}_{11}\left(10-11\right.$ o'clock), and 3 hips at $\mathrm{AA}_{12}$ (11-12 o'clock) position (Figure 4). There was no statistical difference between the mean of AA's of the two genders (males $\mathrm{AA}_{2}: 49^{\circ}$; females $\mathrm{AA}_{2}$ : $\left.47^{\circ}, P=0.4\right)$ at the $\mathrm{AA}_{2}$ segment of the head-neck junction, where the AA's were the highest. No statistically significant difference detected between the two sexes for the mean AA values of all segments (males $\mathrm{AA}_{1-12}: 40^{\circ}$; females $\mathrm{AA}_{1-12}: 38^{\circ}, P=0.3$ ).

The mean age of the patients with cam-type morphology is 15.8 (range : 12-19). Cam-type morphology was not observed in patients younger than 12 years and the prevalence was gradually increased with age (Figure 6).

Pincer-type morphology was seen in 18 of the 102 patients (17.6\%) (31 of the 204 hips, 15.2\%) and 5 patients demonstrated $(27.8 \%)$ unilateral and 13 demonstrated $(72.2 \%)$ bilateral pincer morphology. Pincer-type morphology was more common in females $(7 / 32-21.9 \%)$ than in males $(11 / 70-15.7 \%)$. However, the difference was not statistically significant $(P=0.24)$. The mean age of the patients with pincer-type morphology is 16.2 (range: 1319). Pincer-type morphology was not observed in patients younger than 13 years and the prevalence were gradually increased with age (Figure 7).
Mixed-type morphology was observed in six patients (5.8\%) (10 of the 204 hips, 4.9\%) (5 male and 1 female) and four (66\%) were bilateral. Bilateral mixed-type morphology was detected in only one female.

Triradiate cartilage was closed in $73(72 \%)$ and open in 29 (28\%) out of 102 patients with an average age of 11.2 (range : 9-17), and 16.2 years (range : 11-19), respectively.

Proximal femoral physis was closed in $13(13 \%)$ patients and open in $89(87 \%)$ out of 102 patients with an average age of 17.3 (range : 15-19) and 14.8 (range : 9-19), respectively.

In $24(88 \%)$ patients with cam-type morphology triradiate cartilage was closed, whereas proximal femoral physis was closed only in 3 (11\%) patients.

In all $18(100 \%)$ patients with pincer-type deformity triradiate cartilage was closed, whereas proximal femoral physis was closed only in $3(17 \%)$.

All patients with mix-type deformity had a closed triradiate cartilage $(100 \%)$ and an open proximal femoral physis (100\%) (Table II).

\section{DISCUSSION}

In this study we assessed the prevalence of cam- and pincer-type morphology in asymptomatic 
adolescents and found $17.6 \%$ and $26.5 \%$, respectively. We did not find any statistical difference of cam- and pincer-type morphologies between the two sexes $(P=0.34, P=0.24$, respectively).

The prevalence of FAI morphology among asympthomatic adult population differs from 10 to $38 \%$ in cam- and 10 to $28 \%$ in pincer-type deformity. We found similar prevalence rates as seen in asymptomatic adults but some higher prevalence rates of pincer-type morphology deformity in adult females and cam deformity in adult males has been demonstrated in the literature (17-24). There are only few studies which evaluate the prevalence and features of FAI morphology in pediatric patients. Monazzam et al. investigated the FAI morphology in the pediatric population aged from 2 to 19 years and reported that $10.2 \%$ of males and $2.5 \%$ of females had cam-type morphology and $12.1 \%$ of males and $9.4 \%$ of females had pincer-type morphology (25). We found higher number of patients with cam (26.5\%) and pincer (17.6\%) morphology compared to Monazzam's study, possibly due to the mean age difference between the two studies (10.4 vs 14.8 years) and different AA measurement technique (radial reformated images) of our study.

$\mathrm{Li}$ et al. analyzed 558 adolescents with pelvic CT and found that $16.8 \%, 32.4 \%$, and $6.1 \%$ of the asymptomatic patients had cam-type, pincer-type, and mixed-type morphologies, respectively (26). Not only the mean age of the study populations (14.4 vs 14.8 years) but also the prevalence rates of the cam and mixed-type morphologies were similar compared to our study $(26.5 \%$ and $5.8 \%$ for cam- and mixed-type morphology, respectively). Only the prevalence of pincer morphology was higher than our study (32.4\% vs $17.6 \%)$. We could not find any explanation for this difference in pincer morphology prevalence other than possible morphological variations between different patient populations.

In the same study of Monazzam et. al who performed a retrospective CT-based study and looked for bony radiological parameters associated with FAI in a pediatric and adolescent population and found that CEA and acetabular depth ratio increases whereas Tönnis angle decreases during skeletal development. Furthermore they also noted that AA is decreased until the age of 10 and larger AAs were measured after that year. They concluded that camand pincer-type morphology can occur as early as 10 to 12 years of age (25). We are not able to compare patients younger than 9 year old as our study had limited numbers in this specific patient group but the starting age of cam-type morphology is quite similar with Monazzam's findings (10 vs. 12).

In a cross-sectional cohort study of pediatric population, Carsen et. al assessed the timing of the cam morphology associated with FAI development and whether increased activity level during the period of physeal closure is associated with an increased likelihood of cam morphology development. They concluded that cam morphology is exclusively found in patients with closed femoral physis and increased activity level is a possible risk factor during the period of physeal closure (27). We observed an increase in morphology prevalence among patients with open proximal femoral physis and closed triradiate cartilage.

When searching of the relation of skeletal maturation with development of FAI morphology by assessing physeal closure of the triradiate cartilage and proximal femoral physis we found that the majority of the patients (88\%) with cam-type and all patients $(100 \%)$ with pincer-type morphology had closed triradiate cartilage and $89 \%$ of the patients with cam and $83 \%$ of the patients with pincer-type morphology had open proximal femoral physis.

In a case-control study of Siebenrock et. al, authors proposed that high intensity sports activity during adolescence is associated with a substantial increase in the risk of cam-type impingement. They also concluded that the prevalence of cam morphology in the closed physeal group is higher compared to transitional physeal group (8).

In a recent prospective longitudunal study by van Klij et al. they followed academy male football players for 5-years for the development of camtype morphology using anteroposterior and frog leg views. They found that cam morphology developed in 104 of 142 hips during follow-up and of these 104 hips, cam morphology developed in 86 hips $(82.7 \%)$ with open growth plate and in 18 hips $(17.3 \%)$ with a closed growth plate. Finally they concluded that, cam morphology developed from 12 to 13 
years of age until growth plate closure around 18 years (28).

In our study we found a very high percentage of closed triradiate cartilage in patients who had cam or pincer morphology $(88 \%$ and $100 \%$, respectively). Proximal femoral physis was open in $89 \%$ of the patients with cam morphology and $83 \%$ with pincer deformity. We did not found a cam morphology in patients younger than 12 years and pincer morphology younger than 13 years old. These findings suggest that cam and pincer morphologies likely to develop after triradiate closure and cammorphology develops before the closure of proximal femoral physis.

There are some limitations to our study, since it risk is a retrospective study we were unable to perform the impingement test, and the clinical history of the patients could only be obtained from the medical data provided in hospital information system and for the same reason activity levels and body mass indexes of the patients cannot be factored. Thus, we were not able to evaluate if there was a risk factor especially in physically active adolescents to have cam or pincer deformity. Furthermore our patient population was relatively small.

\section{CONCLUSION}

According to our data, we found similar prevalence rates for cam and pincer morphology in asymptomatic adolescents population as it has previously demonstrated in asymptomatic adults. Cam deformity is located mostly at the anterosuperior portion of the femoral head-neck junction. We also conclude that cam and pincertype morphologies seems to develop during midlate adolescence or after the triradiate cartilage and before the proximal femoral physis closure. Prospective studies designed with larger patient population could be performed for validation of our findings.

\section{REFERENCES}

1. Murray RO. The aetiology of primary osteoarthritis of the hip. Br. J. Radiol. 1965 ; 38 : 810-824.

2. Harris WH. Primary osteoarthritis of the hip : a vanishing diagnosis. J. Rheumatol. 1983 ; Suppl 9 : 64.
3. Solomon L. Studies on the pathogenesis of osteoarthritis of the hip. Trans. Coll. Med. South Africa. 1981 : 104-124.

4. Stulberg SD, Cordell LD, Harris WH, Ramsey PL, MacEwen GD. Unrecognized childhood hip disease : a major cause of idiopathic osteoarthritis of the hip. In : The Hip : Proceedings of the Third Meeting of The Hip Society. St. Louis, MO : CV Mosby ; 1975 : 212-228.

5. Ganz R, Parvizi J, Beck M, Leunig M, Nötzli H, Siebenrock KA. Femoroacetabular impingement : a cause for osteoarthritis of the hip. Clin. Orthop. Relat. Res. 2003 ; 417 : 112-20.

6. Parvizi J, Leunig M, Ganz R. Femoroacetabular impingement. J. Am. Acad. Orthop. Surg. 2007 ; 15 : 561-570.

7. Eijer H, Hogervorst T. Femoroacetabular impingement causes osteoarthritis of the hip by migration and microinstability of the femoral head. Med. Hypotheses. 2017 ; 104 : 93-96.

8. Siebenrock KA, Ferner F, Noble PC, Santore RF, Werlen S, Mamisch TC. The cam-type deformity of the proximal femur arises in childhood in response to vigorous sporting activity. Clin. Orthop. Relat. Res. 2011 ; 469 : 3229-40.

9. Pollard TC, Villar RN, Norton MR, Fern ED, Williams MR, Murray DW. et al. Genetic influences in the aetiology of femoroacetabular impingement: a sibling study. J. Bone Joint Surg. Br. 2010 ; 92 : 209-216.

10. Agricola R, Bessems JH, Ginai AZ, Heijboer MP, van der Heijden RA, Verhaar JA. et al. The development of cam type deformity in adolescent and young male soccer players. Am. J. Sports Med. 2012 ; 40 : 1099-1106.

11. Rakhra KS, Sheikh AM, Allen D, Beaule PE. Comparison of MRI alpha angle measurement planes in femoroacetabular impingement. Clin. Orthop. Relat. Res. $2009 ; 467(3): 660-5$.

12. Notzli HP, Wyss TF, Stoecklin CH, Schmid MR, Treiber $\mathbf{K}$, Hodler J. The contour of the femoral head-neck junction as a predictor for the risk of anterior impingement. J. Bone Joint Surg. Br. 2002 ; 84 : 556-560.

13. Van Houcke J, Yau WP, Yan CH, Huysse W, Dechamps H, Lau WH. et al. Prevalence of radiographic parameters predisposing to femoroacetabular impingement in young asymptomatic Chinese and White subjects. J. Bone Joint Surg. Am. 2015 ; 97 : 310-317.

14. Eijer H, Leunig M, Mahomed MN, Ganz R. Crosstable lateral radiographs for screening of anterior femoral head-neck offset in patients with femoro-acetabular impingement. Hip Int. $2001 ; 11 ; 37-41$.

15. Tonnis D, Heinecke A. Acetabular and femoral anteversion : relationship with osteoarthritis of the hip. J. Bone Joint Surg. Am. $1999 ; 81$ : 1747-1770.

16. Kellinghaus $M$, Schulz R, Vieth V, Schmidt S, Schmeling A. Forensic age estimation in living subjects based on the ossification status of the medial clavicular epiphysis as revealed by thin-slice computed tomography. Int. J. Legal Med. 2010 Jul ; 124(4) : 321-5. 
17. Fraser JL, Sugimoto D, Beng YM, D'Hemecourt P, Straacciolini A. Bony morphology of femoroacetabular impingement in young female dancers and single-sport athletes. Orthop. J. Sports Med. 2017 Aug 10 ; 5(8) : 2325967117723108.

18. Hack K, Di Primio G, Rakhra K, Beaule PE. Prevalence of Cam-type femoroacetabular impingement morphology in asymptomatic volunteers. J. Bone Joint Surg. Am. 2010 ; $92: 2436 \mathrm{e} 44$.

19. Gosvig KK, Jacobsen S, Sonne-Holm S, Gebuhr P. The prevalence of cam-type deformity of the hip joint : a survey of 4151 subjects of the Copenhagen Osteoarthritis Study. Acta Radiol. 2008 ; 49 : 436-441.

20. Kim J, Choi JA, Lee E, Lee KR. Prevalence of Imaging Features on CT Thought to Be Associated With Femoroacetabular Impingement : A Retrospective Analysis of 473 Asymptomatic Adult Hip Joints. AJR Am. J. Roentgenol. 2015 ; 205(1) : W100-5.

21. Polat G, Sahin K, Arzu U, Kendirci AS, Asik M. Prevalence of asymptomatic femoroacetabular impingement in Turkey ; cross-sectional study. Acta Orthop. Traumatol. Turc. $2018 ; 52(01): 49-53$.

22. Van Klij P, Heerey J, Waarsing JH, Agricola R. The prevalence of cam and pincer morphology and its association with development of hip osteoarthritis. $J$. Orthop. Sports Phys. Ther. 2018 Apr ; 48(4) : 230-238.
23. Diesel CV, Ribeiro TA, Coussirat C, Scheidt RB, Macedo CA, Galia CR. Coxa profunda in the diagnosis of pincer-type femoroacetabular 1mpingement and its prevalence in asymptomatic subjects. Bone Joint J. 2015 Apr ; 97-B(4) : 478-83.

24. Ergen FB, Vudalı S, Sarıverdi E, Dolgun A, Aydıngöz Ü. CT Assessment of asymptomatic hip joints for the background of femoroacetabular 1mpingement morphology. Diagn. Interv. Radiol. 2014 ; 20(3) : 271-6.

25. Monazzam S, Bomar JD, Dwek JR, Hosalkar HS, Pennock AT. Development and prevalence of femoroacetabular impingement-associated morphology in a pediatric and adolescent population. Bone Joint J. 2013 ; 95-B : 598-604.

26. Li Y, Helvie P, Mead M, Gagnier J, Hammer MR, Jong N. Prevalence of femoroacetabular impingement morphology in asymptomatic adolescents. J. Pediatr. Orthop. $2017 ; 37: 121-126$.

27. Carsen S, Moroz PJ, Rakhra K, Ward LM, Dunlap H, Hay JA. et al. The Otto Aufranc Award. On the etiology of the cam deformity : a cross-sectional pediatric MRI study. Clin. Orthop. Relat. Res. 2014 ; 472 : 430-436.

28. Van Klij P, Heijboer MP, Ginai AZ, Verhaar JAN, Waarsing JH, Agricola R. Cam morphology in young male football players mostly develops before proximal femoral growth plate closure : a prospective study with 5-year follow-up. Br. J. Sports Med. 2018 ; 0 : 1-8. 